\title{
Energy Efficient Throughput Optimization in Multi-hop Wireless Networks`
}

\author{
Dan $\mathrm{Xu}$ and Xin Liu \\ Computer Science Department, University of California \\ Davis, CA 95616, USA \\ $\{$ xud, liu\}@cs.ucdavis.edu
}

\begin{abstract}
Throughput, fairness, and energy consumption are often conflicting objectives in multi-hop wireless networks. In this paper, we propose the notion of lexicographical maxmin energy efficiency throughput fairness that achieves throughput fairness per unit energy. Compared with maxmin throughput fairness and maxmin time fairness, the proposed scheme allocates more bandwidth to nodes with relay requirements and provides satisfactory bandwidth to nodes far from the sink. We design an optimal bandwidth allocation algorithm to achieve the proposed fairness objective. Simulation results show that the proposed scheme results in more balanced throughput among users when they exhaust energy resources, compared to other fairness schemes.
\end{abstract}

\section{Introduction}

One of the most challenging issues in bandwidth allocation is the conflict between fairness and throughput. In [1], the authors indicate in multi-rate 802.11 MAC, throughput-based fairness degrades network throughput considerably since most channel access time is occupied by low bit rate links. Time-based fairness proposed in 2 allows each user to fairly share time resources, which results in low throughput on low capacity links. In 3, the authors argue in multi-hop WLANs 4, maxmin throughput fairness can improve network throughput without penalizing low capacity users, and maxmin time fairness leads to an even higher network throughput by protecting high bit rate links. In multi-hop networks, some nodes need to serve as routers and relay traffic for other nodes. Routers handle more traffic and thus consume much more energy than descendant nodes. If nodes are energy constrained, such as in sensor networks or when using devices on battery power (e.g., laptops or PDAs), energy consumption needs to be considered in bandwidth allocation among users. This observation motivates the work in this paper.

We consider a multi-hop wireless network where all nodes need to connect to a wired sink or AP (access point) while taking into account energy consumptions. When energy is a constraint, maxmin throughput fairness is unfair to routers

\footnotetext{
* The work was in part supported by NSF through CAREER Award \#0448613 and Grant \#0520126, and by Intel through a gift grant.
} 


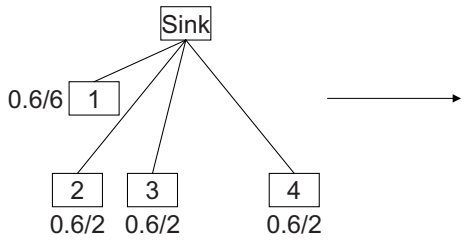

a)

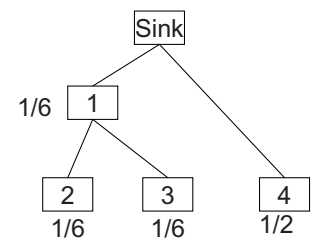

b)

Fig. 1. A example of maxmin throughput fairness bandwidth allocation, in each subfigure, the left side of the slash is the allocated bandwidth and the right side is the node's link rate

because they consume more energy and die much faster than other nodes. Consider the example illustrated by Fig. 11 nodes 2 and 3 choose node 1 as their router since in (a) they both have low bit rates and therefore low throughput if directly connected to the sink. To simplify the example, we assume at each node, transmitting one unit of data costs $2 \mathrm{~J}$ of energy, receiving one unit of data needs $1 \mathrm{~J}$, and no energy is consumed in an idle state. Then in (b) where node 1 is a router, it costs $8 \mathrm{~J}$ per unit time to transmit the same amount of data as node 2 or 3 , which only consume $2 \mathrm{~J}$ per unit time. Therefore, node 1 will have a much shorter lifetime and consequently much less aggregate throughput compared with that of itself in a) and nodes 2 or 3 in b).

On the other hand, maxmin time fairness severely penalizes nodes far away from the sink. This is because, in order to fairly share a router's time, a child node's throughput is about $\frac{1}{2}$ of that allocated to the router. The larger the number of network hops, the less the bandwidth leaf nodes receive. For example, consider a chain topology with 20 nodes and a sink as shown in Fig. 2, Each node chooses the node in front of it as its router. Assume each link's bit rate is $11 \mathrm{Mbps}$. In b), by maxmin time fairness, routers receive much more bandwidth

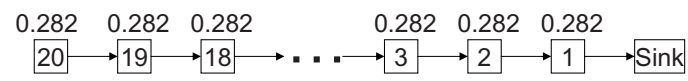

a): Maxmin throughput fairness bandwidth allocation

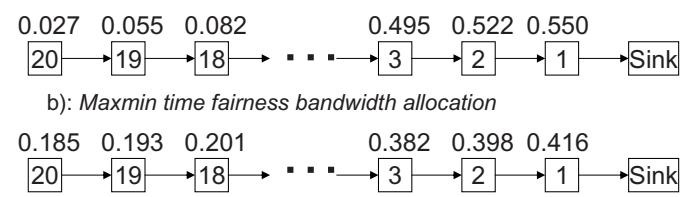

c): Maxmin energy efficiency throughput fairness bandwidth allocation

Fig. 2. In the chain topology, compared with maxmin throughput fairness and maxmin time fairness, maxmin energy efficiency allocates more bandwidth to routers while still giving child nodes relatively high throughput. Here energy consumption for transmit, receive, and idle are $1.9,1.55$, and $0.75 \mathrm{~J} / \mathrm{s}$, respectively. The energy consumption model is based on the measurements reported in [5]. 
compared to their descendants. The leaf node 20 only receives a bandwidth of $27 \mathrm{kbps}$, which is less than $\frac{1}{20}$ of that of node 1 .

Motivated by such limitations, we propose the notion of lexicographical maxmin energy efficiency throughput fairness, which achieves maxmin throughput fairness per unit energy. With this objective, a router is allocated more bandwidth when it consumes more energy per unit time to relay traffic for other nodes. In other words, routers are compensated for providing service for other nodes. Although a router has a shorter lifetime, it can still achieve a high total throughput before using up its battery power. Another benefit is illustrated in Fig. 2, using maxmin energy efficiency throughput fairness, a router can receive higher throughput than its child nodes. Meanwhile, even the leaf node can still receive a satisfactory throughput, which is $185 \mathrm{kbps}$ in the example illustrated by Fig. 2 That is, maxmin energy efficiency throughput fairness provides a better balance among users than both maxmin throughput fairness and maxmin time fairness when energy is the constraint.

Our contributions are as follows. We observe the limitations of maxmin throughput fairness and maxmin time fairness when energy is constrained and propose maxmin energy efficiency throughput fairness that allocates more bandwidth to routers while still not penalizing the bandwidth of children nodes severely. To achieve the objective, we design an iterative optimal bandwidth allocation algorithm. We also propose and implement a maxmin energy fair scheme for comparison. Our results show that maxmin energy efficiency throughput fairness results in more balanced throughput among all nodes when they deplete their energy compared with that of the maxmin throughput fair, maxmin time fair, and maxmin energy fair schemes.

The rest of paper is organized as follows. In Section 2 we describe the network model and state the maxmin energy efficiency throughput fairness. To achieve the defined fairness objective, we design an optimal bandwidth allocation algorithm and validate its correctness in Section 3 . Section 4 is performance evaluation. We present related work in Section 5 and conclusions in Section 6

\section{Formulation}

\subsection{Network Model}

We consider a wireless multi-hop network where each node chooses only one pre-determined first-hop router to be connected to the sink or AP. Therefore, the network topology can be modeled by a tree structure rooted at the sink. In sensor networks or mesh networks, nodes are often static and thus we consider a relatively stable network topology in this paper.

Consider a node $i, a_{i, P_{i}}$ denotes achievable link rate between node $i$ and its parent, which is denoted by $P_{i}$. Notice a sink has no parent node. We use an indicator function $I_{i}$ to denote whether a node is a sink or not, where $I_{i}=1$ if $i$ 
Table 1. Notions of main variables

\begin{tabular}{c|c}
\hline$b_{i}$ & bandwidth of node $i$ \\
$C_{i}$ & the set of child nodes of node $i$ \\
$P_{i}$ & the first hop router of node $i$ \\
$T_{i}$ & the subtree rooted at node $i$ \\
$B_{i}$ & total bandwidth of subtree $T_{i}$ \\
$I_{i}$ & $I_{i}=1$ if $i$ is a non-sink node, $I_{i}=0$ if $i$ is a sink \\
$a_{j, i}$ & actual bit rate from node $j$ to $i$ \\
$e_{t i}$ & energy consumed per unit time in transmit state of node $i$ \\
$e_{r i}$ & energy consumed per unit time in receive state of node $i$ \\
$e_{i i}$ & energy consumed per unit time in idle state of node $i$ \\
$W_{i}$ & workload of $i$, i.e., the sum of time length of receive and transmit state \\
$t_{i i}$ & time length of idle state of node $i$ \\
$E_{i}$ & total energy consumption of node $i$ \\
$e^{2} b_{i}$ & energy efficiency throughput of node $i$ \\
$\mathbf{B}_{i}$ & bandwidth vector of the subtree $T_{i}$ \\
$\mathbf{E}^{2} \mathbf{B}_{i}$ & energy efficiency throughput vector of subtree $T_{i}$ \\
\hline
\end{tabular}

is a non-sink node, $I_{i}=0$ otherwise. Let $C_{i}$ be the set whose first-hop router is $i$ and $C_{i}^{+}$be the set $C_{i} \cup\{i\}$. Let the subtree rooted at node $i$ be $T_{i}$ and $\left|T_{i}\right|$ be the number of nodes in $T_{i}$. Let $b_{i}$ denote the bandwidth allocated to node $i$, and $B_{i}$ be the aggregate bandwidth of subtree $T_{i}$. The notions used in this paper are listed in Table 1 for reference. The time fraction of node $i$ consumed to transmit its own traffic is $\frac{I_{i} b_{i}}{a_{i, P_{i}}}$. Here the time fraction represents the workload added to node $i$. Notice node $i$ also needs to relay traffic for its child node $j \in C_{i}$. The time fraction to relay $j$ 's traffic is $\frac{B_{j}}{a_{j, i}}+\frac{I_{i} B_{j}}{a_{i, P_{i}}}$, while $\frac{B_{j}}{a_{j, i}}$ is the time consumed to receive $j$ 's packets. Since the time fraction at each node can not exceed 1 , the feasible bandwidth allocation condition is given as $\sum_{j \in C_{i}}\left(\frac{B_{j}}{a_{j, i}}+\frac{I_{i} B_{j}}{a_{i, P_{i}}}\right)+\frac{I_{i} b_{i}}{a_{i, P_{i}}} \leq 1$. Similar models are widely used in previous literature [3 8] 12. In such models, the effect of inter-link interference can be ignored or taken into account in the achievable link rate $a_{i, P_{i}}$, as in this article.

In wireless networks, a node may transmit, receive, or stay idle (we ignore the sleeping state in this paper although all studies in this article can be extended to include the sleeping state). Energy consumed in all three states is taken into account in this paper. Let $e_{t i}, e_{r i}$, and $e_{i i}$ be the energy consumptions per unit time of node $i$ in transmit, receive, and idle states, respectively. These parameters are similar for the same kind of wireless terminals. In [5], the authors have measured these parameters for various wireless adapters. The reported values for a 802.11 WLAN module are used in our simulations.

\subsection{Maxmin Energy Efficiency Throughput Fairness}

As illustrated in the example in Fig. 1, a router node consumes much more energy, and therefore has much less overall throughput than its descendant nodes when maxmin throughput fairness is implemented. On the other hand, a node with multiple hops is severely penalized when maxmin time fairness is used. The maxmin energy efficiency throughput fairness is motivated by such limitations and formulated in this section. 
Consider a non-sink node $i$. Its energy consumption per unit time is

$$
E_{i}=\sum_{j \in C_{i}}\left(e_{r i} \frac{B_{j}}{a_{j, i}}+e_{t i} \frac{B_{j}}{a_{i, P_{i}}}\right)+e_{t i} \frac{b_{i}}{a_{i, P_{i}}}+e_{i i}\left(1-\sum_{j \in C_{i}}\left(\frac{B_{j}}{a_{j, i}}+\frac{B_{j}}{a_{i, P_{i}}}\right)-\frac{b_{i}}{a_{i, P_{i}}}\right)
$$

In (11), $1-\sum_{j \in C_{i}}\left(\frac{B_{j}}{a_{j, i}}+\frac{B_{j}}{a_{i, P_{i}}}\right)-\frac{b_{i}}{a_{i, P_{i}}}$ is the idle time of node $i$, denoted as $t_{i i}$, where $t_{i i} \geq 0$.

A non-sink node $i$ consumes $E_{i}$ unit of energy per unit time and achieves a bandwidth of $b_{i}$. We define its energy efficiency bandwidth as $e^{2} b_{i}=\frac{b_{i}}{E_{i}}$, which denotes the bandwidth per energy unit. Suppose there are $n$ non-sink nodes in the network. The energy efficiency bandwidth vector is defined as $\mathbf{E}^{2} \mathbf{B}=\left(\frac{b_{1}}{E_{1}}, \frac{b_{2}}{E_{2}}, \ldots, \frac{b_{n}}{E_{n}}\right)$, where $\frac{b_{1}}{E_{1}} \leq \frac{b_{2}}{E_{2}} \leq \ldots \leq \frac{b_{n}}{E_{n}}$. We give Definition 1 as:

Definition 1(Maxmin Energy Efficiency Throughput Fairness). A feasible bandwidth allocation $\mathbf{B}$ is maxmin energy efficiency throughput fair if its energy efficiency bandwidth vector $\mathbf{E}^{2} \mathbf{B}=\left(\frac{b_{1}}{E_{1}}, \frac{b_{2}}{E_{2}}, \ldots, \frac{b_{n}}{E_{n}}\right)$ is lexicographically equal or larger than that of any other feasible bandwidth allocation.

Informally, a feasible bandwidth allocation is maxmin energy efficiency throughput fair if and only there is no way to increase energy efficiency throughput of any node without decreasing the energy efficiency throughput of some nodes with equal or already less energy efficiency throughput.

The objective of Definition 1 is to provide maxmin fairness of the bandwidth per unit energy. Intuitively, it first allocates the bandwidth per energy unit among all nodes equally. When some nodes are not able to consume the allocated bandwidth within per unit energy, the bandwidth per unit energy can be evenly shared by the rest of the nodes. A desirable property is that a router's energy efficiency throughput is not less than that of its child nodes.

By maxmin energy efficiency throughput fairness, routers that spend more energy can get more bandwidth. If we view the energy as the cost that each node must pay for communications and the bandwidth as the revenue, the more a node contributes, the higher throughput it gets. Although routers have a shorter lifetime, they can still transmit a large amount of data before using up battery power. Therefore, compared with maxmin throughput fairness, the aggregated throughput of a router is significantly improved when it depletes its energy. This mechanism is a good incentive to encourage each node to serve others.

Since maximum energy consumption at node $i$ does not exceed $\frac{e_{t i}}{e_{r j}}$ times of any other node $j$, maximum throughput allocated to a certain router is not excessively higher than its descendants. Compared with maxmin time fairness, the proposed fairness objective provides a satisfactory throughput to leaf nodes, even with a large number of network hops, as shown in Fig. 2. Maxmin energy efficiency throughput fairness provides the fairest overall throughput after each node depletes energy resource. 


\section{Solution}

\subsection{Algorithm Design}

We design an optimal bandwidth allocation algorithm named $\mathrm{E}^{2} \mathrm{TBA}$ (Maxmin Energy Efficiency Throughput Fairness Bandwidth Allocation) to achieve the defined fairness objective. The structure of $\mathrm{E}^{2} \mathrm{TBA}$ is based on the idea of Pump-Drain first proposed in [3]. We use Pump-Drain to convert the problem of maxmin energy efficiency throughput fairness bandwidth allocation, which can be modeled as a serial Quasi-optimization problem, to a simper problem of solving a non-linear equation set. Then we give an approximate algorithm to obtain the solution numerically.

$\mathrm{E}^{2} \mathrm{TFA}$ runs in the recursive and distributed way: a sink initializes $\mathrm{E}^{2} \mathrm{TFA}$, then $\mathrm{E}^{2} \mathrm{TFA}$ recursively calls $\mathrm{E}^{2} \mathrm{TFA}$ in the up-bottom order. After $\mathrm{E}^{2} \mathrm{TFA}$ execution at a node $j \in C_{i}$ returns, node $i$ will perform Pump-Drain within $T_{i}$ to achieve maxmin energy efficiency throughput fairness among all the nodes in $T_{i}$.

To perform Pump-Drain, each node $i$ maintains the following information that is locally reported by its children nodes. After performing Pump-Drain, node $i$ also reports the information to its parent node.

- The bandwidth assigned to each node $k$ in $T_{i}$, namely $b_{k}$.

- The distinct amounts of energy efficiency bandwidth assigned to $T_{i}$, which are sorted in the array $\lambda_{i}$ in a non-decreasing order. $\left|\lambda_{i}\right|$ is the up-to-date number of the elements of $\lambda_{i}$.

- In array $\xi_{i}$, the $k$ th element $\xi_{i}[k]$ is a set of nodes whose energy efficiency bandwidth is equal to $\lambda_{i}[k]$.

- The structure of $T_{i}$.

The details of $\mathrm{E}^{2} \mathrm{TFA}$ are as follows.

Pump: Initially, the energy efficiency bandwidth of each node is zero(Bandwidth of each node is set to zero). After the execution of $\mathrm{E}^{2} \mathrm{TFA}$ returns from a child node of $T_{i}$. Pump is executed at node $i$ in the following steps.

I. If node $i$ is a sink, let $b_{i}$ keep the value of 0 and there is no need to perform Pump. However, Drain maybe needed since bandwidth of its children nodes may make $i$ overloaded. If node $i$ is a non-sink node, Pump goes to Step II.

II. For a non-sink node $i$, after the execution of $\mathrm{E}^{2} \mathrm{TFA}$ at $j \in C_{i}$ returns, the bandwidth allocation within $T_{j}$ is maxmin energy efficiency throughput fair and node $j$ is saturated. The aggregated bandwidth of $T_{j}$ is $B_{j}$, whose time share at node $i$ is $\frac{B_{j}}{a_{j, i}}+\frac{B_{j}}{a_{i, P_{i}}}$. The energy efficiency bandwidth of node $j$ is $e^{2} b_{j}=\frac{b_{j}}{E_{j}}$. At node $i$, the time fraction left to support its own bandwidth is 
$1-\sum_{j \in C_{i}}\left(\frac{B_{j}}{a_{j, i}}+\frac{B_{j}}{a_{i, P_{i}}}\right)$, then the bandwidth of node $i$ that can be supported is:

$$
\phi=\left(1-\sum_{j \in C_{i}}\left(\frac{B_{j}}{a_{j, i}}+\frac{B_{j}}{a_{i, P_{i}}}\right)\right) a_{i, P_{i}}
$$

With the bandwidth of $\phi$, there is no idle time at node $i$, and the corresponding energy efficiency bandwidth of node $i$ is

$$
e^{2} b_{i}=\frac{\phi}{E_{i}}=\frac{\phi}{\sum_{j \in C_{i}}\left(\frac{e_{r i}}{a_{j, i}}+\frac{e_{t i}}{a_{i, P_{i}}}\right) B_{j}+\frac{e_{t i}}{a_{i, P_{i}}} \phi}
$$

When $\frac{\phi}{\sum_{j \in C_{i}}\left(\frac{e_{r i}}{a_{j, i}}+\frac{e_{t i}}{a_{i, P_{i}}}\right) B_{j}+\frac{e_{t i}}{a_{i, P_{i}}} \phi} \geq \lambda_{i}\left[\left|\lambda_{i}\right|\right]$, i.e., energy efficiency bandwidth of node $i$ is larger than that of any other node in $T_{i}$, let $b_{i}=\phi$. Pump stops and there is no need to perform Drain. Otherwise, Pump goes to Step III.

III. When $\frac{\phi}{\sum_{j \in C_{i}}\left(\frac{e_{r i}}{a_{j, i}}+\frac{e_{t i}}{a_{i, P_{i}}}\right) B_{j}+\frac{e_{t i}}{a_{i, P_{i}}} \phi}<\lambda_{i}\left[\left|\lambda_{i}\right|\right]$, we let the energy efficiency bandwidth of node $i$ equal to $\lambda_{i}\left[\left|\lambda_{i}\right|\right]$. Node $i$ gets the bandwidth $\varphi$ by solving the following equation,

$$
\lambda_{i}\left[\left|\lambda_{i}\right|\right]=\frac{\varphi}{\sum_{j \in C_{i}}\left(\frac{e_{r i}}{a_{j, i}}+\frac{e_{t i}}{a_{i, P_{i}}}\right) B_{j}+\frac{e_{t i}}{a_{i, P_{i}}} \varphi},
$$

Then $\varphi=\frac{\lambda_{i}\left[\left|\lambda_{i}\right|\right] \sum_{j \in C_{i}}\left(\frac{e_{r i}}{a_{j, i}}+\frac{e_{t i}}{a_{i, P_{i}}}\right) B_{j}}{1-\lambda_{i}\left[\left|\lambda_{i}\right|\right] \frac{e_{t i}}{a_{i, P_{i}}}}$. Now node $i$ also has the largest energy efficiency bandwidth among all the nodes in $T_{i}$. Since $\varphi>\phi$, node $i$ must be overloaded, Pump stops and Drain is needed to decrease the bandwidth of $T_{i}$ to make the bandwidth allocation feasible.

Drain: The objective of Drain is to decease the bandwidth of each node in $\xi_{i}\left[\left|\lambda_{i}\right|\right]$ to make the workload of node $i$ feasible, i.e., let $W_{i}=1$. Meanwhile, the energy efficiency bandwidth of each node should still stay the same. However, since in $\xi_{i}\left[\left|\lambda_{i}\right|\right]$, deceasing the bandwidth of a node will probably result in the decrease of energy consumption of itself and all the ancestor nodes, decreasing the bandwidth of each node while keeping their energy efficiency bandwidth the same is a non-trivial job. In $\xi_{i}\left[\left|\lambda_{i}\right|\right]$, there are three kinds of nodes: first, the nodes without any children in $\xi_{i}\left[\left|\lambda_{i}\right|\right]$, we use $k$ to denote them; the second is those with children in $\xi_{i}\left[\left|\lambda_{i}\right|\right]$, we denote these with $l$; the last is node $i$ itself. Then mathematically, the above problem can be solved by the equation set (5).

The equation in \{\}$^{*}$ appears in (5) only when $i$ is a non-sink node. Notice in (5) the idle time and idle energy consumption of node $i$ is 0 , since $i$ is saturated before Drain is completed. In (5), the bandwidth of each node is decreased to make the workload of node $i$ equal to 1, while keeping energy efficiency bandwidth the same, which is denoted by $\eta$. When $W_{i}=1$ is satisfied, if $\eta \geq \lambda_{i}\left[\left|\lambda_{i}\right|-1\right]$, then Drain is done. If $\eta<\lambda_{i}\left[\left|\lambda_{i}\right|-1\right]$, simply decreasing 
the bandwidth of nodes in $\xi_{i}\left[\left|\lambda_{i}\right|\right]$ is not enough, the bandwidth of nodes in $\xi_{i}\left[\left|\lambda_{i}\right|-1\right]$ should also be decreased. Then Drain is performed by two steps: first, decrease the bandwidth of each node in $\xi_{i}\left[\left|\lambda_{i}\right|\right]$ to make their energy efficiency bandwidth equal to $\lambda_{i}\left[\left|\lambda_{i}\right|-1\right]$, which can be done through replacing $\eta$ by $\lambda_{i}\left[\left|\lambda_{i}\right|-1\right]$ in (5) and deleting the final equation; second, combine the set $\xi_{i}\left[\left|\lambda_{i}\right|\right]$ and $\xi_{i}\left[\left|\lambda_{i}\right|-1\right]$ and do the same operation described by (5). If at this time the current $\lambda_{i}\left[\left|\lambda_{i}\right|\right]$ is still smaller than the current $\lambda_{i}\left[\left|\lambda_{i}\right|-1\right]$, repeat the above two steps.

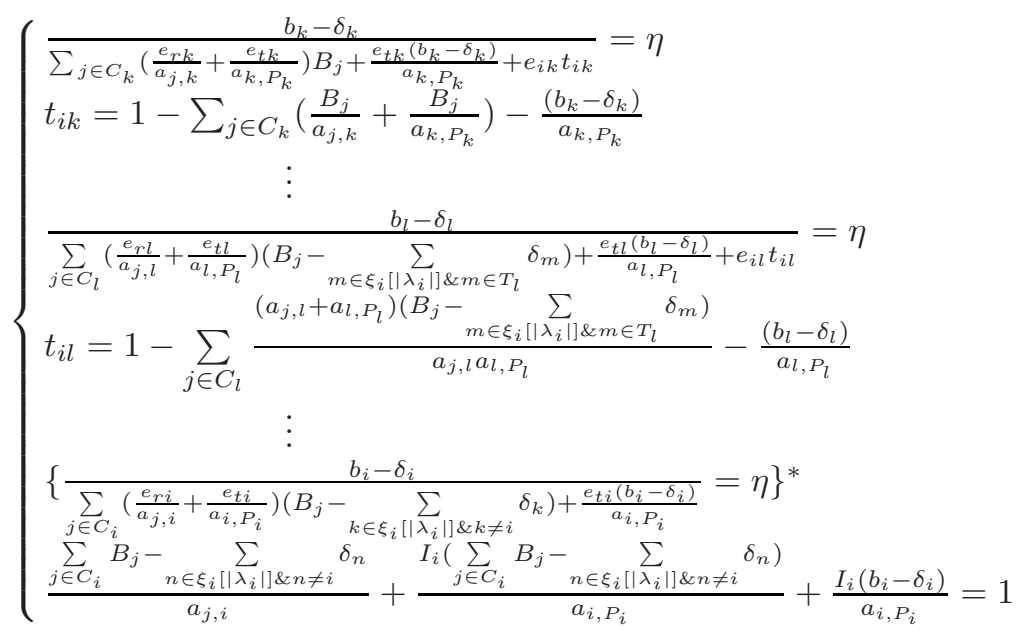

(5) is a non-linear equation set which definitely has an optimal solution. However, numerically, there is only an approximate algorithm to solve (5). Here we design an efficient distributed algorithm to perform Drain which can get approximate optimal numerical results.

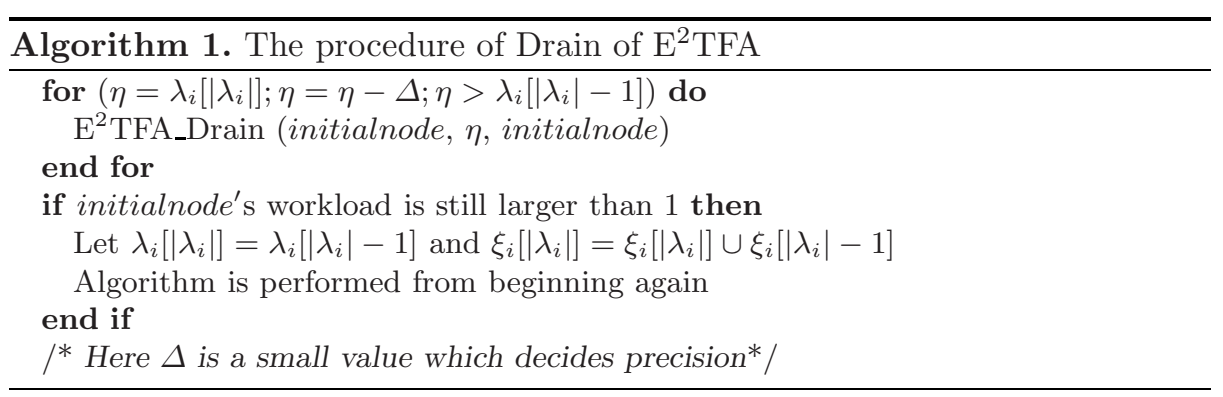

\subsection{Correctness Validation}

Proposition 1. $\mathrm{E}^{2} \mathrm{TFA}$ achieves maxmin energy efficiency throughput fairness. Proof is available at [6]. 


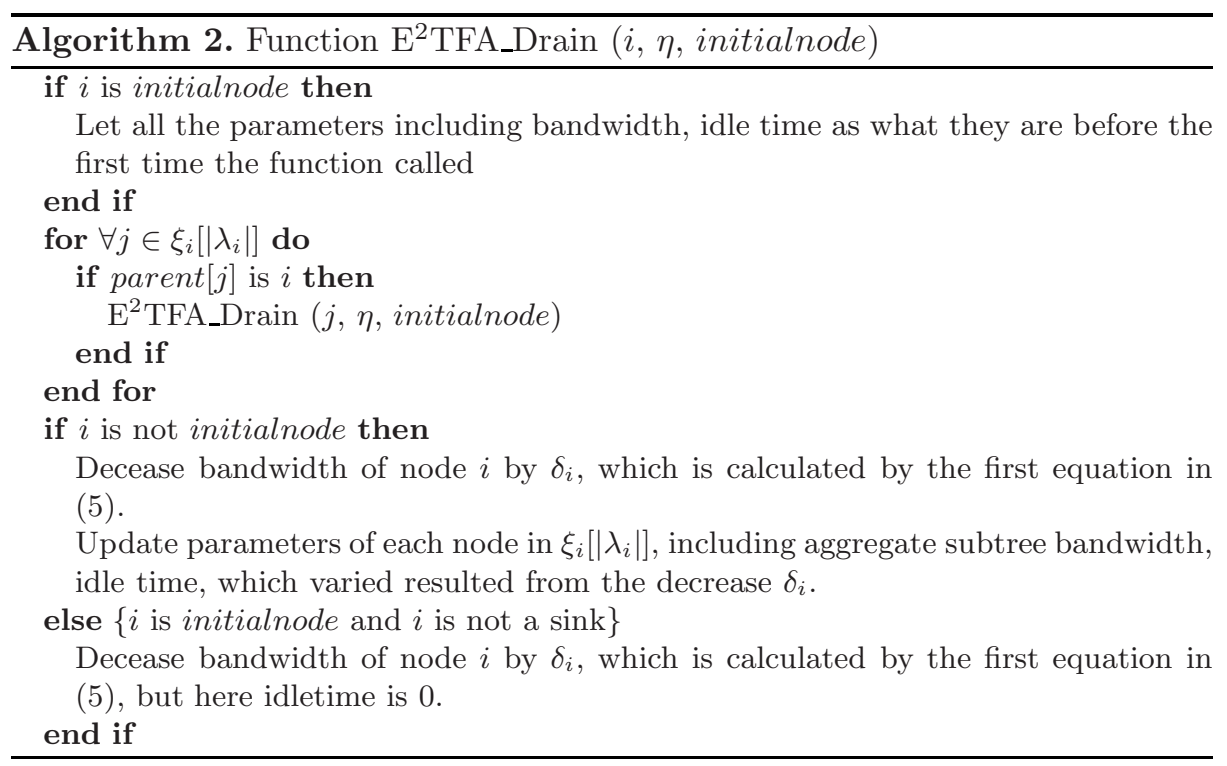

\section{Evaluation}

In this section, we evaluate the performance of $\mathrm{E}^{2} \mathrm{TFA}$ and compare it with other fairness schemes including maxmin throughput fairness bandwidth allocation(MMFA), maxmin time fairness bandwidth allocation(MTFA) and maxmin energy fairness bandwidth allocation(MEFA). MMFA and MTFA have been studied in 3. We propose MEFA for comparison. In MEFA, each router's energy resource is fairly shared by all its descendants and itself. The idea of MEFA is similar with that of MTFA. Therefore, MEFA should have similar performance to MTFA.

In $\mathrm{E}^{2} \mathrm{TFA}$, routes are predetermined. Because finding an optimal routing that yields best throughput is NP-hard, we implement two heuristic schemes. The first is the tree construction algorithm proposed in [3], which can iteratively improve throughput. We call it ITCA here. ITCA provides good performance for networks with a small number of hops but works slowly when the number of hops increases. Therefore, we also deploy a shortest distance routing (SDR) scheme, where a node chooses its first-hop router which is the nearest one among the nodes that have a shorter distance with the sink. SDR is efficient in large wireless networks.

In the simulation, we consider an area of $150 \mathrm{~m} * 150 \mathrm{~m}$. The link bit rate is determined as follows: it is $11 \mathrm{Mb} / \mathrm{s}$ when the distance between a transmitter and its receiver is smaller than $50 \mathrm{~m}, 5.5 \mathrm{Mb} / \mathrm{s}$ when distance is smaller than $80 \mathrm{~m}$, $2 \mathrm{Mbps}$ when smaller than $120 \mathrm{~m}$, and $1 \mathrm{Mbps}$ when larger than $120 \mathrm{~m}$. Unit energy consumptions in the transmit, receive, and idle states are $1.9 \mathrm{~J} / \mathrm{s}, 1.55 \mathrm{~J} / \mathrm{s}$, and $0.75 \mathrm{~J} / \mathrm{s}$, respectively [5]. We conduct simulations in the following two scenarios. 
- Scenario A: 4 sinks are located at each corner and 30 nodes are randomly located in the square. ITCA is used.

- Scenario B: 1 sink is located at a corner and 25 nodes are randomly located in the square. SDR is used.

First, we study the aggregate throughput of each node when it depletes energy. The initial energy of each node is set to be 100J. We compare MMFA, MTFA, MEFA and $E^{2}$ TFA in both Scenario A and B. When a node runs out its energy, it quits the networks and the four schemes are executed for the rest of the nodes. As showed in Fig. 3, in both scenarios, $\mathrm{E}^{2} \mathrm{TFA}$ provides the fairest aggregate throughput among all nodes. In Scenario A, using MMFA, routers only transmit a small amount of data before they deplete their energy resources. While in B, the aggregate throughput of leaf nodes in MTFA and MEFA is very low, since in Scenario B there is only a sink, the number of network hops is large, throughput of leaf nodes is severely penalized by MEFA and MTFA. Although leaf nodes have a longer lifetime, their aggregate throughput is still very low. Notice that routers have higher aggregate throughput, throughput, and energy efficiency throughput in MTFA and MEFA, which is contrary to that in MMFA.

In Fig. 4, in both scenarios, MMFA results in fairest throughput $\left(b_{i}\right)$ among all the nodes. On the other hand, MTFA allocates much higher throughput to routers than children nodes. In Scenario B, throughput of leaf nodes allocated by MTFA is only $\frac{1}{4}$ of that given by MMFA. However, in the same scenario,

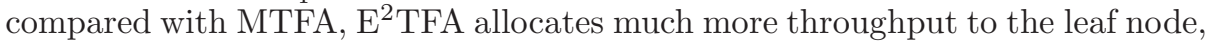
which is about 3 times more than that of MTFA. Meanwhile it still allocates similar high throughput to a router. In both scenarios, $\mathrm{E}^{2} \mathrm{TFA}$ gives higher throughput to routers without severely penalizing any node.

In Fig. 5, we show that $\mathrm{E}^{2} \mathrm{TFA}$ achieves the fairest throughput per unit energy $\left(\frac{b_{i}}{E_{i}}\right)$, which is the objective of $\mathrm{E}^{2} \mathrm{TFA}$. By MMFA, children nodes have smaller energy consumption and therefore have high energy efficiency throughput. MTFA and MEFA gives routers excessively high throughput, which result in a higher energy efficiency throughput for routers although they have relatively higher energy consumption. This aslo illustrates that MTFA is severely biased towards nodes near the sink.
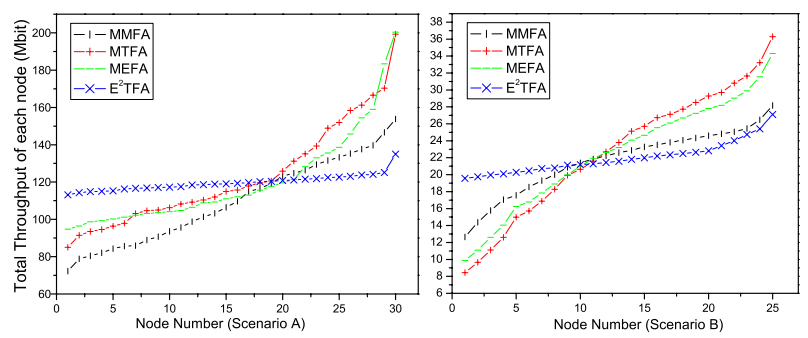

Fig. 3. Each node's aggregate throughput 


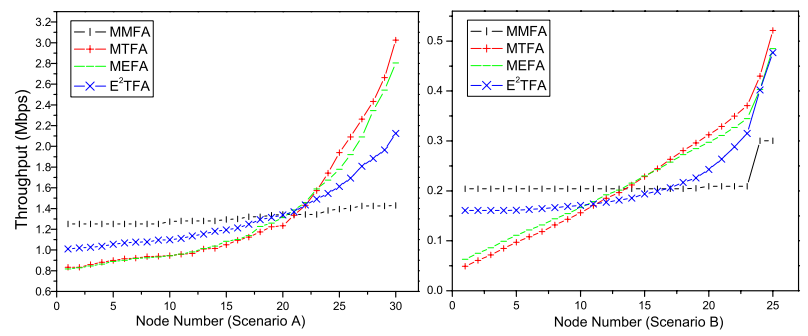

Fig. 4. Throughput of each node

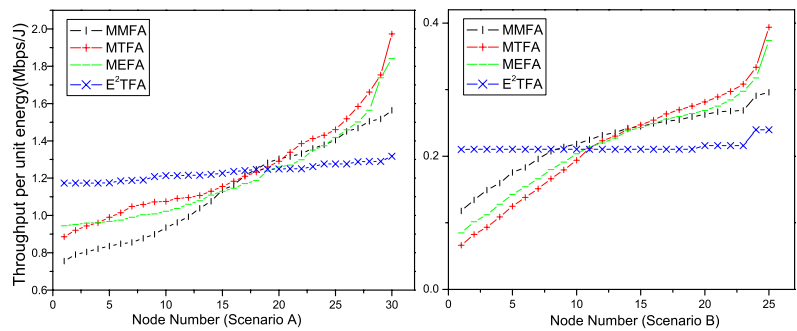

Fig. 5. Energy efficiency throughput

\section{Related Work}

In [3, the authors consider both maxmin throughput fairness and maxmin time fairness in multi-hop WLANs and design an optimal bandwidth allocation algorithm for each objective. In [8] 9] [10], the authors study MAC layer scheduling or bandwidth allocation for ad hoc networks. In [12, the authors study maxmin fairness bandwidth allocation in multi-AP single-hop WLANs through association control. Since the problem is NP-hard, algorithms to determine user-AP associations are proposed that attain near-optimal maxmin fairness. In [13], the authors study maximum and maxmin fairness bandwidth allocation in multichannel wireless mesh networks. All the above works do not consider energy constraints. In [14, the authors consider maxmin fairness rate allocation in sensor networks. Flow split is allowed and thus the problem can be solved by a serial LP with lifetime constraints.

\section{Conclusion}

In this paper, we study throughput fairness and optimization in energy-constrained multi-hop wireless networks. We observe that maxmin throughput fairness biases against routers with heavier traffic while maxmin time fairness biases against nodes with more hops to the sink. Motivated by such observations, we propose the notion 
of lexicographical maxmin energy efficiency throughput fairness with the following properties. First, the proposed fairness objective allocates more bandwidth to routers that relay packets for others and therefore encourages them to serve others; second, the throughput discrepancy between routers and their descendants is bounded. Therefore, leaf nodes can still receive satisfactory throughput even in a large network; third, by combining energy consumption and throughput, our scheme results in the most balanced aggregate throughput when all nodes use up the energy resources. We develop a distributed algorithm to achieve the above objective and validate its advantages through extensive simulations.

\section{References}

1. M. Heusse, F. Rousseau, G. Berger-Sabbatel, and A. Duda, "Performance anomaly of 802.11b," in IEEE Infocom, 2003.

2. G. Tan and J. Guttag, "Time-based fairness improves performance in multi-rate wireless lans," in USENIX Annual Technical Conference, 2004.

3. Q. Dong, S. Banerjee, B. Liu, "Throughput Optimization and Fair Bandwidth Allocation in Multi-Hop Wireless LANs," in IEEE Infocom, 2006.

4. S. Lee, S. Banerjee, and B. Bhattacharjee, "The case for a multi-hop wireless local area network," in IEEE Infocom, 2004.

5. O.Kasten, "Energy Consumption," available at http://www.inf.ethz.ch/personal/ kasten/research/bathtub/energy_consumption.html

6. Dan Xu and Xin Liu, "Energy Efficient Throughput Optimization in Multi-hop Wireless Networks," http://www.cs.ucdavis.edu/liu/.

7. T. Nandagopal, T.-E. Kim, X. Guo, and V. Bharghavan, "Achieving mac layer fairness in wireless packet networks," in ACM MobiCom, 2000.

8. L. Tassiulas and S. Sarkar, "Maxmin fair scheduling in wireless networks," in IEEE Infocom, 2002.

9. S. Lu, H. Luo and V. Bharghavan, A new model for packet scheduling in multihop wireless networks,in Proceedings of ACM MobiCom, 2000.

10. X. L. Huang and B. Bensaou, "On max-min fairness and scheduling in wireless ad-hoc networks: Analytical framework and implementation," in Proceedings of in ACM MobiHoc, 2001.

11. A. Penttinen, I. Koutsopoulos and L. Tassiulas, "Low-complexity distributed fair scheduling for wireless multi-hop networks," in IEEE Wiopt, 2005.

12. Y. Bejerano, S.-J. Han, and L. E. Li, "Fairness and load balancing in wireless lans using association control," in Proceedings of ACM MobiCom, 2004.

13. J. Tang, G. Xue, W. Zhang, "Maximum throughput and fair bandwidth allocation in multi-channel wireless mesh networks," in IEEE Infocom, 2006.

14. Y. Hou, Y. Shi, H. Sherali, "Rate Allocation in Wireless Sensor Networks with Network Lifetime Requirement," in Proceedings of ACM MobiHoc, 2004. 